\title{
Fibrinogen-Fibrin Conversion
}

\author{
THE MECHANISM OF FIBRIN-POLYMER FORMATION IN SOLUTION
}

\author{
Gerald F. SMITH \\ Lilly Research Laboratories, Division of Eli Lilly and Company, Indianapolis, IN 46206, U.S.A.
}

(Received 24 January 1979)

\begin{abstract}
The fibrin polymers formed in solution during the earliest phase of the fibrinogen-fibrin conversion are shown to be stable soluble molecules at $\mathrm{pH} 7.4$ and $0.15 \mathrm{M}-$ or $0.3 \mathrm{M}-\mathrm{NaCl}$. The various sequential soluble fibrin polymers produced from the fibrinogen-thrombin reaction can be observed by gel chromatography and can be isolated for characterization. The mechanism of fibrin polymerization proposed from the present studies suggests that the initial event is the thrombin activation at only one of the $A \alpha$-chains in fibrinogen. The resulting highly reactive intermediate is the true fibrin monomer and it rapidly, and irreversibly, self-associates to form the stable fibrin dimer $\left(s_{20 . w}=12 S\right)$. Fibrin dimer possesses the $N$-terminal pattern alanine/glycine/tyrosine $(1: 1: 2)$ per 340000 molecular weight, and possesses the chain structure $\left.[(\alpha) A \alpha)(B \beta)_{2}(\gamma)_{2}\right]_{2}$. The fibrin dimer is a soluble inert molecule, but additional thrombin activation of its remaining intact $A \alpha$-chains leads to new associations into larger inert soluble fibrin polymers. In this manner progressively larger fibrin oligomers are constructed with thrombin continually in control of the process because of the necessity to repeatedly re-activate the various fibrin polymers in solution. The inert character of the soluble fibrin polymers can be explained by the reciprocal alignment of the associating molecules, which mutually consumes their active surfaces and leaves an intact $A \alpha$-chain at either end of each fibrin oligomer. The soluble fibrin polymers will proceed to further association only if thrombin activates these remaining $A \alpha$-chains, otherwise the fibrin molecules are stable indefinitely. The intermolecular associations within the soluble fibrin polymers are essentially irreversible under these nearly physiological conditions. However, the bonding is not covalent. This mechanism accounts for the clinical observations of stable fibrinogen-derived polymers in the plasma from patients undergoing thrombotic processes. Since it is shown that the intermediate fibrin polymers, themselves, are stable soluble molecules, it is no longer necessary, nor warranted, to invoke hypothetical 'fibrinogen-fibrin complexes' to explain observations of fibrin solubility.
\end{abstract}

The conversion of fibrinogen into fibrin is one of the most important biochemical reactions, since blood clotting is a fundamental part of the homoeostatic system, and also because fibrin formation plays important roles in many pathological processes, e.g., overt thrombosis, cancer, diabetes and inflammatory diseases (Zimmerman, 1976; Wessler, 1977).

Abbreviations used: Bz-Phe-Val-Arg-pNA, benzoylphenylalanylvalylarginine $p$-nitroanilide; SDS, sodium dodecyl sulphate; Temed, $N N N^{\prime} N^{\prime}$-tetramethyl-ethylenediamine; the three pairs of fibrinogen chains are designated singly as $\mathrm{A} \alpha, \mathrm{B} \beta$ and $\gamma$; when the fibrinopeptides have been removed from the $A \alpha$-and $B \beta$-chains, the chain designations become $\alpha$ and $\beta$ respectively; thus the three pairs of fibrinogen chains, as a molecule, are designated as $(A \alpha)_{2}(B \beta)_{2}(\gamma)_{2}$, whereas fully thrombin-proteolysed fibrinogen is designated as $(\alpha)_{2}(\beta)_{2}(\gamma)_{2} ; \mathrm{NpGdBzO}^{-}$, p-nitrophenylguanidinobenzoate.
Accordingly, the chemical reactions that produce fibrin are of great importance and have been the subject of many multidisciplinary studies since the 1940 's.

One aspect of fibrin formation that has remained poorly understood is the earliest stage in the process, wherein the thrombin-activated fibrinogen molecules polymerize in solution. During this earliest stage the intermediate fibrin polymers are produced, which are the precursor polymers for the distinctly later fibrin products the fibrils and fibres.

This problem has been approached indirectly, in the past, because of the general belief that fibrin moleccules are insoluble under physiological conditions (Shainoff \& Page, 1960), because of their presumed uncontrolled tendency to aggregate into insoluble networks. Accordingly, the intermediate fibrin polymers have been presumed to represent 
unstable transient intermediates that could be studied in solution only in the presence of polymerization inhibitors, in the presence of chaotropic agents, or hydrogen-bond-breaking solvents or in solutions of non-physiological $\mathrm{pH}$ values (for reviews see Scheraga \& Laskowski, 1957; Doolittle, 1973).

The purpose of the present work was to elucidate the mechanism(s) of fibrin polymerization in solution by directly studying the fibrinogen-thrombin reaction under nearly physiological conditions $\left(37^{\circ} \mathrm{C}, \mathrm{pH} 7.4\right.$ and in $0.15 \mathrm{M}$ - or $0.3 \mathrm{M}-\mathrm{NaCl}$ ). The experimental aims were to use gel chromatography to observe and to fractionate the various soluble molecular populations evolving during the fibrinogen-fibrin conversion and to physically and chemically characterize the soluble fibrin oligomers. The premise for this approach was that the soluble products of the polymerization phase of the fibrinogen-fibrin conversion might not be labile under these conditions, rather they might be sufficiently stable for direct study in solution. This premise was based on findings by Smith \& Bang (1972), working with aqueous buffered clotting systems, and on observations by other workers on blood plasma-clotting systems (Kierulf \& Abildgaard, 1971; von Hugo \& Graeff, 1973; Ly \& Jakobsen, 1975) and on blood plasma from patients (Alkjaersig et al., 1975; Edgar et al., 1977). Each of these studies had strongly suggested that polymeric forms of fibrin do exist in solution under physiological conditions.

\section{Materials and Methods}

\section{Human fibrinogen}

Fibrinogen was isolated from a 2 litre pool of human citrate-treated plasma (not yet past the 'expiration date'), purchased from a local hospital blood bank, by combination of the methods of Kazal et al. (1963, 1964), Mosesson \& Sherry (1966) and Mosesson (1962). Only variations from these procedures are described below. Plastic containers were used for all operations. Solid 6-aminohexanoic acid (Sigma) was added to the plasma, to a concentration of $0.1 \mathrm{M}$, immediately on pooling. After centrifugation to remove cell debris, the plasma was treated three times by $\mathrm{BaSO}_{4}$ adsorption (J. T. Baker powder) at successive concentrations of $90 \mathrm{~g} /$ litre, $90 \mathrm{~g} /$ litre and $45 \mathrm{~g} /$ litre. After each $1 \mathrm{~h}$ adsorption period and centrifugation at $5000 \mathrm{~g}$, the plasma was tested for thromboplastin-clotting time $(0.1 \mathrm{ml}$ of plasma plus $0.1 \mathrm{ml}$ of Dade thromboplastin reagent) and recalcification-clotting time $[0.1 \mathrm{ml}$ of plasma + $0.1 \mathrm{ml}$ of $\mathrm{CaCl}_{2}(0.02 \mathrm{M}$, containing $0.2 \mathrm{M}$-6-aminohexanoic acid)]. After the third $\mathrm{BaSO}_{4}$ treatment, the recalcification-clotting time was infinite (greater than 4 days, when observation was ceased) and the thromboplastin-clotting time was $>10 \mathrm{~h}$. This plasma was then treated by the methods cited in the above references with Matheson glycine crystals, maintaining the 6-aminohexanoic acid concentration at $0.1 \mathrm{M}$ in all of the solutions during all operations, to isolate the fibrinogen as fractions I-1, I-2, I-8 and I-9. Only fibrinogen fraction I-2, the so-called lowsolubility, or normal, fibrinogen molecular population, was used in the experimental work described in the present paper. The isolated fibrinogen I-2 was dialysed against $0.06 \mathrm{M}-\mathrm{Tris} / 0.3 \mathrm{M}-\mathrm{NaCl}(\mathrm{pH} 7.4)$ and stored frozen. The fibrinogen was free from plasmin, plasmin inhibitor, thrombin inhibitor, heparin cofactor, plasminogen and factor XIII, as judged by the absence of these activities in the following assays: plasmin, no thrombin-clot lysis over an extended time period; plasminogen, no thrombin-clot lysis with urokinase and no fibrinogen lysis with urokinase as judged by immunoelectrophoresis and gel chromatography; plasmin inhibitor, no ability to change standard plasmin activity on fibrin-plate assay; thrombin inhibitor, no ability to inhibit thrombin hydrolysis of Bz-Phe-Val-Arg-pNA ; heparin cofactor, no ability to increase the inherent heparin thrombininhibition activity in the presence of heparin (Smith, 1977); factor XIII, no $5 \mathrm{M}$-urea-insoluble fibrin and covalently bound chains in fibrin on SDS/dithiothreitol $/ 10 \%$ polyacrylamide-gel electrophoresis. Fraction I-9 was used as a molecular-weight standard (275000) for gel chromatography.

\section{Thrombin}

Bovine thrombin was purified from Parke Davis topical thrombin by the methods of Rasmussen (1955) and McCoy et al. (1974) and was assayed by plasmaclotting kinetics against N.I.H. reference human thrombin (lot 3-B) and found to have an activity of 2200 N.I.H. units/mg (Smith \& Craft, 1976). The thrombin was judged to be free of $\alpha_{2}$-antithrombin by SDS/polyacrylamide-gel electrophoresis. In the experiments described in the present paper the same results were obtained by using either purified thrombin or the crude topical thrombin. Thrombin was dissolved in $0.06 \mathrm{M}-\mathrm{Tris} / 0.3 \mathrm{M}-\mathrm{NaCl}(\mathrm{pH} 7.4)$.

\section{Gel chromatography}

Agarose-gel columns were prepared from Bio-Gel A (Bio-Rad). Descending eluent flow was regulated with a Mariotte flask. U.v. monitoring was performed with an ISCO model UA-2 analyser with $0.5 \mathrm{~cm}$ path-flow cells. When a series of elution patterns are compared in the text, the family of curves were obtained on the same column. Void volumes were determined by using Dextran Blue 2000 (Pharmacia). In all gel-chromatographic experiments the eluting buffer was $0.06 \mathrm{M}-\mathrm{Tris} / 0.15 \mathrm{M}-\mathrm{NaCl}(\mathrm{pH} 7.4)$ or $0.06 \mathrm{M}-\mathrm{Tris} / 0.3 \mathrm{M}-\mathrm{NaCl}(\mathrm{pH} 7.4)$. The operating temperature was ambient. For guanidine- or SDS-gel chromatography, the gel columns were prepared 
with the desired concentration of the respective reagents and the eluting buffer was, likewise, made up to the same concentration of SDS or guanidine.

Molecular-size determination by gel chromatography

By using a single column $(0.9 \mathrm{~cm} \times 28 \mathrm{~cm})$ of Bio-Gel A-5 m (100-200 mesh, lot 11961), fibrinogen I-2, fibrinogen I-9 and fibrin dimer were carefully analysed for their gel-elution characteristics. Samples were applied in $0.3 \mathrm{ml}$ volumes $(0.3 \mathrm{mg})$ and effluents were measured with a micro-graduated cylinder. Triplicate analyses of the peak elution volumes were repeatable to within $3 \%$. The following elutionvolume/void-volume ratios were obtained: fibrinogen I-9 (mol.wt. 270000), 1.81; fibrinogen I-2 (mol.wt. 340000), 1.75; fibrin dimer (mol.wt. 680000), 1.58. These data, when plotted as elution-volume/voidvolume ratio against log (molecular weight), yielded a straight line.

\section{Polyacrylamide/SDS-gel discontinuous electropho-} resis

The method of Davis (1964) was modified to give gels containing $5 \%$ acrylamide $/ 0.13 \%$ bisacrylamide/ $0.02 \% \quad(\mathrm{v} / \mathrm{v})$ Temed $/ 0.090 \% \quad\left(\mathrm{NH}_{4}\right)_{2} \mathrm{~S}_{2} \mathrm{O}_{8} / 0.10 \%$ $\mathrm{SDS} / 0.27 \mathrm{M}-\mathrm{Tris} / 0.043 \mathrm{M}-\mathrm{HCl}$ (pH 8.9) or $10 \%$ acrylamide $/ 0.26 \%$ bisacrylamide $/ 0.027 \%$ (v/v) Temed/ $0.073 \% \quad\left(\mathrm{NH}_{4}\right)_{2} \mathrm{~S}_{2} \mathrm{O}_{8} / 0.10 \%$ SDS $/ 0.355 \mathrm{M}$-Tris/ $0.057 \mathrm{M}-\mathrm{HCl}(\mathrm{pH} 8.9)$. The running buffer was $0.1 \% \mathrm{SDS} / 0.035 \mathrm{~m}$-glycine/0.005 $\mathrm{m}$-Tris (pH8.3). Reagents were from Bio-Rad. Voltage was $250 \mathrm{~V}$ ( $5 \mathrm{~mA}$ per tube) and duration was 1-1.5 h. Gel columns had the dimensions $0.5 \mathrm{~cm} \times 13 \mathrm{~cm}$.

\section{Sedimentation-velocity analysis}

Sedimentation experiments were performed with a Beckman model E ultracentrifuge with the sedimentation boundaries measured by u.v. optics. The data were obtained from simultaneous analysis of sample groups in a multi-compartmented rotor (AN-G): the temperature was $23.0^{\circ} \mathrm{C}$, the angular velocity was $40000 \mathrm{rev} . / \mathrm{min}$ and the magnification

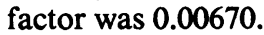

\section{$N$-Terminal amino acid analysis}

This technique was based on the Edman phenylthiohydantoin method. The procedure used was developed especially for fibrinogen/fibrin analysis. The experimental details, control data and critical evaluation of the technique (errors of $4 \%$ or less are to be expected) were reported by Smith \& Murray $(1968,1970)$ and Murray \& Smith (1968).

\section{Pooling fibrin fractions for $\mathrm{N}$-terminal analysis}

Soluble fibrin-polymer fractions from preparative gel chromatography were pooled and the soluble fibrin was precipitated completely by adding $\left(\mathrm{NH}_{4}\right)_{2} \mathrm{SO}_{4}$ to $35 \%$ saturation. The precipitates were centrifuged into pellets and washed with $35 \%$ saturated $\left(\mathrm{NH}_{4}\right)_{2} \mathrm{SO}_{4}$, then were washed with $50 \%$ acetone (MCB spectral grade). The wash liquors were aspirated from the centrifuged pellets and the products frozen and dried over $\mathrm{P}_{2} \mathrm{O}_{5}$ in vacuo. This procedure was repeated until four such preparative experiments were pooled together to yield sufficient dry protein for $N$-terminal amino acid analysis.

\section{Results}

\section{Fibrinogen-fibrin conversion in solution}

The dynamic molecular polymerizations occurring during the thrombin-induced fibrinogen-fibrin conversion (from incipient thrombin action until gelation) are demonstrated by the analytical-gelchromatography curves in Fig. 1. The experiment described in Fig. 1 involved the treatment of human fibrinogen $[4 \mathrm{mg} / \mathrm{ml}$ in $0.06 \mathrm{M}-\mathrm{Tris} / 0.3 \mathrm{M}-\mathrm{NaCl}$

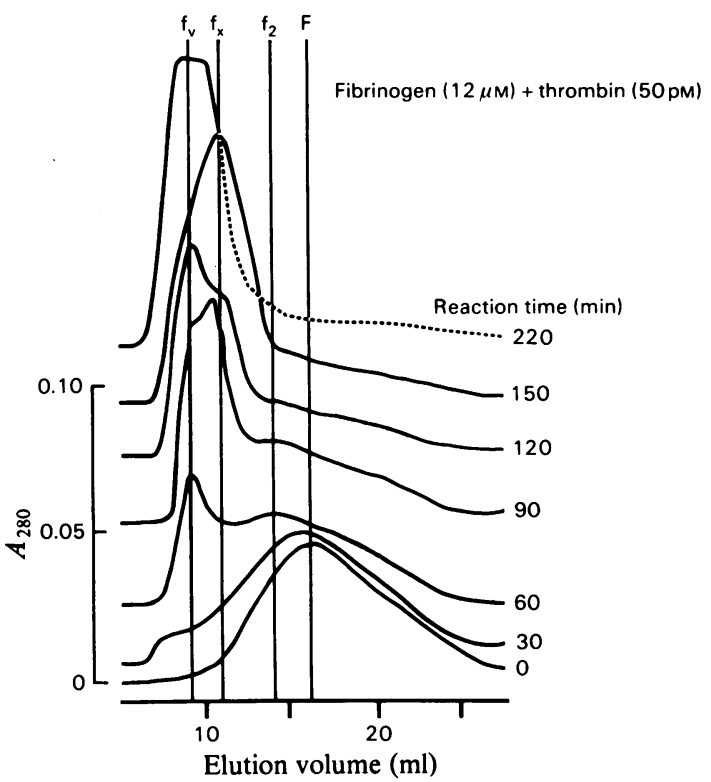

Fig. 1. Gel chromatography of the fibrinogen-thrombin reaction stopped at the desired times and indicated as reaction times

Reaction solvent and column eluent was $0.06 \mathrm{M}$ Tris/0.3 $\mathrm{M}-\mathrm{NaCl}$ (pH 7.4). Eluents were monitored by $A_{230}$ in the range $0-0.1$. Reaction mixture was $4 \mathrm{mg}$ of human fibrinogen $/ \mathrm{ml}$ and 0.005 N.I.H. units of thrombin per $\mathrm{ml}$, at $37^{\circ} \mathrm{C}$. Portions of $0.2 \mathrm{ml}$ $(0.8 \mathrm{mg})$ were applied to a column $(0.9 \mathrm{~cm} \times 29 \mathrm{~cm})$ of A-50m agarose gel (lot 120581; 50-100 mesh). The flow rate was $0.47 \mathrm{ml} / \mathrm{min}$. The $t=0$ curve was from a portion of the fibrinogen before thrombin was added. Curves are drawn with baselines displaced. Vertical lines indicate zones of elution for $F$ (fibrinogen), $f_{2}$ (fibrin dimer), $f_{x}$ (larger soluble fibrin polymers) and $f_{v}$ (void-volume soluble fibrin polymers). 
(pH 7.4)] with bovine thrombin (final concentration 0.005 N.I.H. units $/ \mathrm{ml}$ ) at $37^{\circ} \mathrm{C}$. Portions were removed from this reaction mixture at various times and treated with $\mathrm{NpGdBzO}^{-}$to inhibit thrombin, and then were analysed on an agarose $(\mathrm{A}-50 \mathrm{~m}) \mathrm{gel}$ column (void volume $9.0 \mathrm{ml}$ ). The control fibrinogenelution pattern $(0 \mathrm{~min})$ showed a peak at $16 \mathrm{ml}$. By $60 \mathrm{~min}$ reaction time the fibrin dimer $\left(\mathrm{f}_{2}\right)$ was distinguished $(14 \mathrm{ml})$ and a significant population of molecules eluted in the void volume. At least one other molecular population was resolved at $90 \mathrm{~min}$ reaction time ( $f_{x}$ polymers) eluting at about $11 \mathrm{ml}$. By reaction times of $120 \mathrm{~min}$ and $150 \mathrm{~min}$ the build-up of the void volume eluting fibrin polymers (labelled $f_{\text {vold }}$ ) and the $f_{x}$ populations occurred at the expense of the fibrinogen $(F)$ and fibrin dimer $\left(f_{2}\right)$ populations. From $150 \mathrm{~min}$ reaction time until $220 \mathrm{~min}$ reaction time the void volume soluble fibrin polymers were steadily increasing at the expense of all other molecular populations in the reaction mixture. By $240 \mathrm{~min}$ reaction time the reaction mixture had begun to form a soft gel that increased in firmness, with time, to form a firm clot.

The reaction mixture was too viscous to sample after $220 \mathrm{~min}$ reaction time. Therefore the $t=220 \mathrm{~min}$ curve represents the molecular state of affairs in the fibrinogen-fibrin conversion just before gelation (or clotting). Just before coagulation in this unstirred reaction essentially all the fibrinogen was activated and incorporated into soluble fibrin polymers.

The portions taken at the various times for analysis in Fig. 1 all showed complete recovery of total protein during gel chromatography, showing that no fibrin precipitation occurred at any point in the reaction before the gel point $(240-270 \mathrm{~min})$. Hence the sequence of analytical curves in Fig. 1 represents the total distribution of molecular species during the fibrinogen-fibrin conversion at $\mathrm{pH} 7.4,0.15-0.3 \mathrm{M}-$ $\mathrm{NaCl}$ and $37^{\circ} \mathrm{C}$. For data in $0.15 \mathrm{M}-\mathrm{NaCl}$ buffer see below.

Experiments such as that shown in Fig. 1 were performed in which portions of the reaction mixtures, having been treated with $\mathrm{NpGdBzO}^{-}$(or hirudin), were left at room temperature for $24 \mathrm{~h}$, or were frozen and stored for 1-4 days to be thawed and analysed. In all cases the gel-elution patterns of the portions were not changed. Thus on stopping the thrombin enzymic catalysis in a thrombin-fibrinogen reaction mixture, the various ongoing individual polymerization reactions immediately cease and the molecular array of products is 'frozen'. This implies that the polymerization reactions between activated species are extremely fast and that the rate-controlling step and the driving force of the whole process is the continual thrombin activation of all the species in solution. When thrombin is added back to any of the stopped reactions the mixtures proceed to form fibrin clots.
When using the same experimental approach to examine the fibrinogen-thrombin reaction in $0.15 \mathrm{M}$ $\mathrm{NaCl}$ buffer, there were no discernible differences from the experiments conducted in the $0.3 \mathrm{M}-\mathrm{NaCl}$ buffer (the same soluble fibrin oligomers were formed in the same sequence with the same properties). The overall reaction rate is about 1.3 times faster in the lower ionic-strength medium. This difference in total reaction rate in the two ionic-strength environments is simply due to ionic effects on thrombin catalytic activity, since the same rate difference was found when measuring the thrombin hydrolysis of Bz-PheVal-Arg-pNA at 0.15 and 0.30 ionic strengths. This is consistent with previous observations that thrombin-proteolytic activity on a variety of substrates is similarly affected by similar variations in ionic strength (Sherry et al., 1954; Blombäck, 1958; Svendsen et al., 1972).

\section{Preparative fractionation of soluble fibrin polymers}

The remarkable stability of the various soluble fibrin polymers (see above) suggested that they could be isolated by preparative gel chromatography. The experiment described in Fig. 2 demonstrates the production and fractionation of soluble fibrin polymers. This fibrinogen-thrombin reaction was allowed to progress to a point where the various classes of soluble oligomers would show measurable populations, e.g., to a point represented by the $t=60 \mathrm{~min}$ curve of Fig. 1, and the reaction was terminated at that point. A small portion was taken for analytical gel chromatography to confirm that the spectrum of soluble fibrin products was, indeed, similar to the $t=60 \mathrm{~min}$ curve of Fig. 1. The whole stopped-reaction mixture was fractionated on a preparative column of Bio-Gel A-5m (Fig. $2 a$ ). The void volume of the column in Fig. 2(a) was about $50 \mathrm{ml}$ and control fibrinogen was eluted from the column as a single symmetrical peak (broken curve) at about $90 \mathrm{ml}$. The preparative elution pattern (solid curve) shows that, indeed, a spectrum of soluble molecules of various molecular weights was fractionated in this experiment, ranging from fibrinogen-sized molecules $(80-100 \mathrm{ml}$ elution) to much larger molecules eluting at the void volume. The fraction numbers $5,10,15,20,25,30,35$ and 40 , as indicated in Fig. 2(a), were selected for analytical study. Fig. 2(b) shows the analytical gel chromatography of these fractions ( $1 \mathrm{ml}$ portions).

In Fig. $2(b)$ the elution of control fibrinogen is shown by the broken curve, a single symmetrical peak at $11.8 \mathrm{ml}$ elution volume (the void volume of the column used was about $7.0 \mathrm{ml}$ ). The elution curves for fractions 30,35 and 40 were all identical and only that from fraction $\mathbf{3 0}$ is shown; it indicates that the only protein in this fraction is exactly the same size as fibrinogen. Fraction 25 has enough of a larger component, which we can designate as fibrin 
(a)

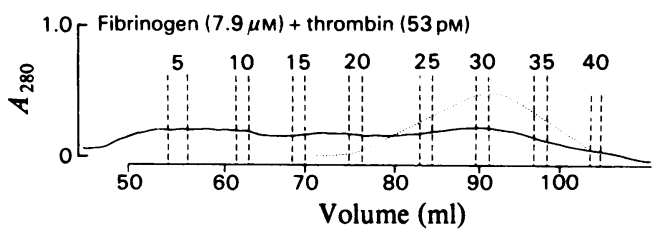

(b)

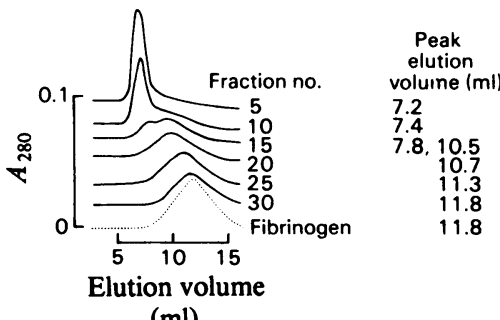

(ml)

Fig. 2 Separation and isolation of stable soluble fibrin polymers

(a) shows preparative gel chromatography (solid curve) of fibrinogen-thrombin reaction stopped after $45 \mathrm{~min}$. The reaction mixture was $2.7 \mathrm{mg}$ of human fibrinogen $/ \mathrm{ml}$ and 0.0053 N.I.H. units of thrombin $/ \mathrm{ml}$ with reaction conditions identical with those in Fig. 1. The entire stopped reaction $(10.8 \mathrm{mg})$ was applied to a column of A-5m agarose (lot 11961, 100-200 mesh; flow rate $0.46 \mathrm{ml} / \mathrm{min}$ ). Absorbance was measured in the range $0-1.0$, and fractions were collected in tubes for $3 \mathrm{~min}$ each fraction. The broken elution curve is control fibrinogen, in the same amount, from this preparatory column $(2.5 \mathrm{~cm} \times 26 \mathrm{~cm})$. The fraction tubes selected for further analysis are indicated in Fig. 2(a) with the vertical broken lines showing the section of the elution-volume pattern that each fraction tube encompassed. (b) shows analytical gel chromatography $[0.9 \mathrm{~cm} \times 28 \mathrm{~cm}$ column of A-5m agarose (lot $11961 ; 100-200 \mathrm{mesh})]$ of fraction tubes from Fig. 2(a). Samples were $1.0 \mathrm{ml}$ $(0.3-0.4 \mathrm{mg})$, flow rate was $0.45 \mathrm{ml} / \mathrm{min}$ and absorbance was measured in the range $0-0.1$. The solid curves, with baselines elevated, show the elution patterns of the fraction tubes, as indicated by numerals. The broken elution curve is from control fibrinogen $(0.27 \mathrm{mg})$ on the same column. Chart speed was $10.16 \mathrm{~cm} / \mathrm{h}$.

dimer (see below), so that its elution pattern has moved slightly to the left. Fraction 20 shows this larger component (fibrin dimer) as a distinct separated peak from fibrinogen and also distinctly separated from the larger soluble fibrin polymers. Fraction 15 shows an elution pattern suggesting it contains fibrin dimer and a larger soluble fibrin component, which we can designate as $f_{x}$. Fraction 10 has an elution pattern suggesting significant populations of $f_{x}$ fibrin polymers and larger void volume polymers $\left(f_{\text {void }}\right)$, whereas the fibrin dimer population is very low. Fraction 5 is composed of only void-volume polymers and some $f_{x}$ fibrin molecules, but has no fibrin dimer population.

The analytical-gel chromatograms of Fig. $2(b)$ were obtained immediately on isolation of the various fractions from the preparative chromatography. However, these same fractions were then frozen and rechromatographed after thawing. The resulting curves were identical with those of Fig. 2(b). These thawed fractions were left overnight at room temperature for a third series of analytical chromatography, and again the gel-elution patterns were identical with those of Fig. 2(b). Therefore the oligomeric soluble fibrin polymers demonstrate extreme stability, tending to neither dissociate nor to further aggregate when fractionated into various molecular-sized populations. The results described in Fig. 2 are also obtained when performing the fibrinogen-thrombin reaction at 0.15 ionic strength, except that the total reaction rate is slightly faster (see above).

\section{Sedimentation coefficients $\left(s_{20, w}\right)$ of soluble fibrin- polymer fractions}

Ultracentrifugal analysis of fractions from the experiment in Fig. 2 and from a number of other similar experiments with A-15m agarose preparative gel chromatography yielded the $s_{20 \text {,w }}$ values $(0.1-$ $0.5 \mathrm{mg}$ of protein $/ \mathrm{ml}$ ): fibrinogen (e.g. fraction 39 , Fig. 2), 7s; fibrin dimer (e.g. fraction 15, Fig. 2), 12S; $f_{x}$ polymers (e.g. fraction 10 , Fig. 2 ), $17 S ; f_{\text {vold }}$ polymers (e.g. fraction 5, Fig. 2), 23S.

\section{Non-covalent nature of the intermolecular bonding within soluble fibrin polymers}

The extremely strong intermolecular bonding that allows the soluble fibrin polymers to be isolated, and to be diluted (during gel chromatography) without detecting dissociation, was shown to be non-covalent in character. Fig. 3, for example, shows the $10 \%$ polyacrylamide-gel electrophoresis in the presence of $0.1 \% \mathrm{SDS}$, intramolecular disulphide bonds having been broken by dithiothreitol. The electrophoretic gels in Fig. 3 show control fibrinogen and three fractions of soluble fibrin polymers from a preparative experiment exactly like that in Fig. 2(a) (the fibrin samples correspond to fractions 5,10 and 15 in Fig. 2a). The three soluble fibrin samples show only bands similar to the $A \alpha-, B \beta$ - and $\gamma$-chains of the fibrinogen sample. Absolutely no covalent linkage exists between or among any of the chains, as covalent bonding would have produced slower moving protein bands in the gels (this type of analysis is commonly utilized to discriminate between cross-linked and non-crosslinked chains in fibrin). Many soluble fibrin fractions have been analysed in this fashion, including all of the fractions discussed in Figs. 2(a) and 2(b). In all cases, all of the fibrin polymers isolated and characterized ( $f_{\text {vold }}, f_{x}$ and $f_{2}$ dimer) analyse exactly as the 


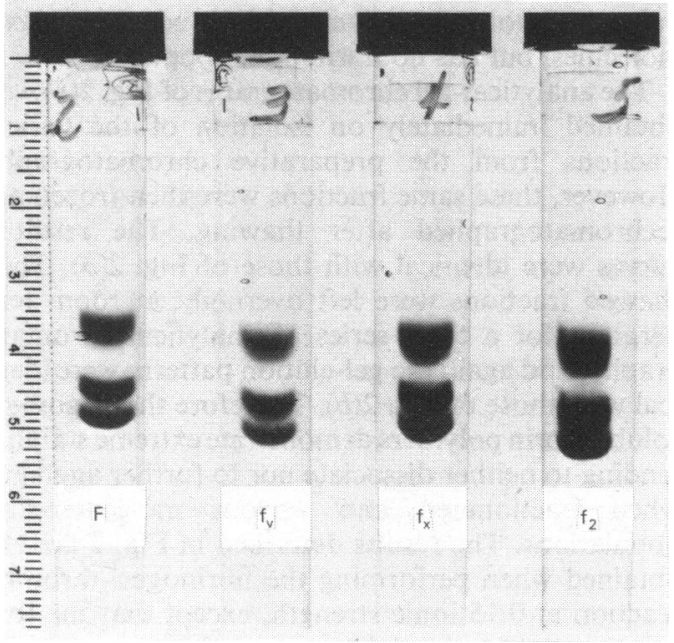

Fig. 3. $10 \%$ polyacrylamide/SDS-gel discontinuous electrophoresis with disulphide bond-reduced samples Samples were isolated fractions from a stopped fibrinogen-thrombin reaction identical with the conditions and preparative chromatography described in the legend to Fig. 2(a). Gels shown are: F, control fibrinogen; $f_{v}$, soluble fibrin fraction no. 5 $(8 \mu \mathrm{g}) ; f_{x}$, fraction no. $10(14 \mu \mathrm{g}) ; f_{2}$, fraction no. 15 (fibrin dimer) $(16 \mu \mathrm{g})$. Electrophoretic movement was downward (toward the anode). The bands, from top to bottom, are $\mathbf{A} \alpha$ - (or $\alpha-$ ), B $\beta$ - and $\gamma$-chains. Sample $(50 \mu \mathrm{l}, 0.1-0.3 \mathrm{~g} /$ litre $)$ were incubated with $10 \mu \mathrm{l}$ of $6 \% \mathrm{SDS}$ and $10 \mu \mathrm{l}$ of $4 \%$ dithiothreitol for $15 \mathrm{~min}$ at $37^{\circ} \mathrm{C} ; 20 \mu \mathrm{l}$ of $100 \%(\mathrm{w} / \mathrm{v})$ sucrose added and the whole mixture applied to $10 \%$ polyacrylamide/SDS gels, prepared as described in the Materials and Methods section.

samples of Fig. 3, and no covalent bonding can be demonstrated.

The intermolecular bonds in the soluble fibrin polymers are broken by SDS without disrupting the disulphide bonds. This was shown by electrophoretically examining all the fibrin fractions discussed above after incubating the fibrin polymer fractions with $2 \%$ SDS. In every instance, the pattern produced on $5 \%$ polyacrylamide-gel electrophoresis, with $0.1 \%$ SDS, was identical with control fibrinogen; all detergent-treated polymers, $f_{\text {vold }}, f_{x}$ and $f_{2}$, were no longer oligomeric, but migrated as bands identical with fibrinogen.

Guanidine was also found to dissociate the soluble fibrin oligomers. $f_{\text {vold }}$ and $f_{x}$ soluble fibrin fractions were made $3.4 \mathrm{M}$ with respect to guanidine and then analysed on an analytical gel A-50m agarose column in $3.4 \mathrm{M}$-guanidine buffer. The soluble fibrin polymers in the $3.4 \mathrm{M}$-guanidine mixture eluted from the $3.4 \mathrm{M}$-guanidine A-50m column exactly like control fibrinogen.
Hence the extremely strong intermolecular bonding within the soluble fibrin oligomers is not covalent in nature, but results from forces disruptible by guanidine and by detergents.

\section{$N$-Terminal amino acids in soluble fibrin oligomers}

Of the six chains in fibrinogen, four are thrombinsusceptible, namely, the two $A \alpha$-chains $(N$-terminating in alanine) and the two B $\beta$-chains ( $N$-terminating in 5-oxoproline). Each thrombin-activation event proteolytically cleaves an arginylglycine bond, producing a new glycine $N$-terminal group, whereas the original $N$-terminal residue leaves as part of the activation peptide. The two fibrinogen $\gamma$-chains have $N$-terminal tyrosine residues. Phenyl isothiocyanate will not react with 5-oxoprolyl end-groups. Hence fibrinogen yields the pattern alanine/tyrosine residues (2:2) per 340000 molecular weight; if one A-fibrinopeptide is cleaved, the resulting molecule yields alanine/glycine/tyrosine residues $(1: 1: 2)$ per 340000 molecular weight; if both A-fibrinopeptides and both B-fibrinopeptides are cleaved, the resulting molecule yields glycine/tyrosine residues $(4: 2)$ per 340000 molecular weight.

Since it was now possible to isolate stable fibrin oligomers from the fibrinogen-fibrin conversion, the extent of thrombin activation could be determined by quantitatively measuring the $N$-terminal amino acids in the isolated soluble fibrin-polymer fractions. Thus preparative fibrinogen-fibrin-conversion reactions were designed to isolate sufficient quantities

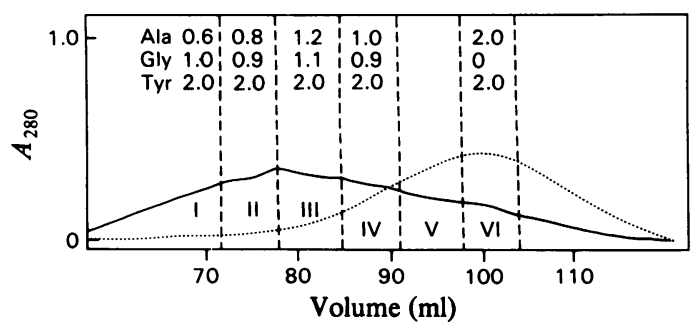

Fig. 4. N-Terminal amino acid analysis of soluble fibrin polymers

The fibrinogen-thrombin reaction was stopped after $40 \mathrm{~min}$ and fractionated into pools for quantitative $N$-terminal analysis. The solid curve shows the preparative gel chromatography of $25 \mathrm{mg}$ of the stopped-reaction mixture. The broken elution curve is of control fibrinogen from this A-5m column $(2.5 \mathrm{~cm} \times 30 \mathrm{~cm}$; lot 11961$)$. The vertical broken lines show the zones pooled together for precipitation of the protein. The $N$-terminal data are given in the Figure as residues $/ 340000$ molecular weight. The sample sizes used for analysis were: I, $12 \mathrm{mg}$; II, $10 \mathrm{mg}$; III, $15 \mathrm{mg}$; IV, $14 \mathrm{mg}$; VI, $7 \mathrm{mg}$. The concentrations of fibrinogen and thrombin were $21 \mu \mathrm{M}$ and 140pM respectively. 
of fibrin dimer, $f_{\text {vold }}$ polymers and the fibrinogensized molecular species, for $N$-terminal analysis. Humanfibrinogen $(3.5 \mathrm{ml} ; 7.1 \mathrm{mg} / \mathrm{ml})$ was treated with thrombin $(0.014$ N.I.H. units $/ \mathrm{ml})$ at $\mathrm{pH} 7.4$ and $37^{\circ} \mathrm{C}$ for $40 \mathrm{~min}$, the reaction being stopped with $\mathrm{NpGdBzO}^{-}$. The extent of reaction was shown to be between the $t=30 \mathrm{~min}$ and the $t=60 \mathrm{~min}$ curves of Fig. 1. The stopped-fibrinogen-fibrin-conversion mixture was fractionated on a preparation column of A-5 $\mathrm{m}$ agarose. The preparative-gel-elution pattern is shown in Fig. 4 as the solid curve (the elution of $25 \mathrm{mg}$ of control fibrinogen from that column is shown by the broken curve). The pooled fractions for $\mathrm{N}$-terminal amino acid analysis are indicated by the vertical broken lines and the $N$-terminal amino acids found in the pooled fibrin oligomeric fractions are indicated in their respective elution region. The $N$-terminal data are given in residues $/ 340000$ molecular weight (the molecular size of fibrinogen).

The elution characteristics of this preparatory column (Fig. 4) are as follows. Fibrinogen is eluted from 85 to $115 \mathrm{ml}$, fibrin dimer is eluted from 70 to $90 \mathrm{ml}$ and $f_{\text {void }}$ polymers (and $f_{x}$ polymers) are eluted from 55 to $75 \mathrm{ml}$. The eluent flow was $0.53 \mathrm{ml} / \mathrm{min}$.

The pooled fraction VI, containing only fibrinogensized protein, showed an $N$-terminal amino acid content of two alanine and two tyrosine residues $/ \mathrm{mol}$; only unactivated fibrinogen molecules are present in this pool and fibrin monomers (which would be detectable by their $N$-terminal glycine) are not present. This shows that fibrin monomers that are generated by thrombin action are too reactive to remain 'monomeric' in solution, the 'monomers' very rapidly, and irreversibly, dimerizing to the stable fibrin dimer.

The elution region for fibrin dimer on the preparatory column of Fig. 4 is spanned by the three pooled fractions II, III and IV. On the basis of analytical gel chromatography fibrin dimer was the exclusive component of zones III and IV and was the main component of zone II (with some overlap with $\mathrm{f}_{\mathrm{x}}$ and $\mathrm{f}_{\text {void }}$ polymers). The $N$-terminal amino acid pattern demonstrated by the fibrin dimer population in each pooled fraction is alanine/glycine/tyrosine residues $(1: 1: 2)$ per mol of fibrinogen subunit (mol.wt. 340000). The chemical composition of fibrin dimer is defined therefore as the dimer of two minimally thrombin-activated fibrinogen molecules (each having lost one A-fibrinopeptide); i.e. $(\gamma)_{2}(\mathrm{~B} \beta)_{2}(\mathrm{~A} \alpha)(\alpha)-(\alpha)(\mathrm{A} \alpha)(\mathrm{B} \beta)_{2}(\gamma)_{2}$.

The fibrin polymers in the fraction I of Fig. 4 were all $\left(f_{\text {void }}-f_{x}\right)$-sized species; no fibrin dimer (or other smaller molecule) was present. The $N$-terminal amino acids found in the $f_{v o l d}-f_{x}$ polymers of fraction I, Fig. 4, had alanine/glycine/tyrosine residues $(0.6: 1.0: 20)$ per 340000 molecular weight. It has been shown that the $N$-terminal analytical technique used should produce data with an error of no more than $10 \%$ with fibrinogen and fibrin (Smith, 1968; Smith \& Murray, 1970). In spite of the quantitative uncertainty that is apparent in this particular result, valuable qualitative deductions can be made. The $N$-terminal pattern shows significant $N$-terminal alanine (uncleaved A-fibrinopeptides) in these inert $\mathrm{f}_{\text {vold }}$ fibrin polymers. This demonstrates that the large soluble fibrin molecules, although they are inert to further spontaneous polymerization (they have used up the active surfaces revealed by thrombin), possess $A \alpha$-chains, which can later be activated by thrombin.

\section{Discussion}

A direct study of the earliest phase of the fibrinogenfibrin conversion, fibrin polymerization in solution, has been reported in the present paper. It was demonstrated that, indeed, this process mediated by thrombin under nearly physiological conditions (pH7.4 and ionic strength 0.3 or 0.15 ) can be experimentally monitored without altering the reaction environment from the beginning of thrombin proteolysis until gelation of the reaction mixture.

The experiments of Figs. 1 and 2 are positive proof that the intermediate fibrin polymers are soluble stable (indeed, inert) molecules. These stable soluble fibrin polymers are the precursor polymers for the distinctly later fibrin products the fibrils and fibres. The intermolecular associations within the soluble fibrin polymers (including the fibrin dimer) were found to be essentially irreversible at $\mathrm{pH} 7.4$ and ionic strength 0.15 or 0.3 . However, it was demonstrated that the intermolecular bonds within the soluble fibrin oligomers are not covalent.

The fact that the soluble fibrin oligomer formation was not effected by changing the ionic-strength environment from 0.15 to 0.30 (except for the total reaction rate as governed by small changes in thrombin activity) contrasts with the significant effects produced in fibrin fibril structure by these same subtle changes in ionic strength (Shen et al., 1977). This contrast suggests that the mechanism whereby the activated fibrinogen molecules associate into soluble fibrin polymers is different from the mechanism(s) whereby the soluble fibrin polymers associate to form fibrils and fibres. In support of this difference in association mechanisms is the recent finding (G. F. Smith \& J. L. Sundboom, unpublished work) that $\mathrm{Ca}^{2+}(0.0025 \mathrm{M})$ in experiments identical with that of Fig. $1(0.15 \mathrm{M}-\mathrm{NaCl})$ does not alter the results, but that the resulting clot formed is very opaque (in contrast with the clear clot formed without added $\left.\mathrm{Ca}^{2+}\right)$. Thus the effect of $\mathrm{Ca}^{2+}$ on fibrin-clot opacity is not mediated during soluble fibrin polymerization; rather, the effect operates during fibril or fibre formation. Manyakov et al. (1977) have suggested that many, if not most, of the interactions 
involved in fibril formation occur beyond the gelation point (possibly involving subunit rearrangements).

It was also shown that the entire fibrin-polymerization process is kinetically controlled by thrombin activity. As long as a thrombin activity persists, or if thrombin is reintroduced into stoppedreaction mixtures, the fibrinogen-fibrin conversion will be driven forward, but stopping the thrombin activity will arrest the process.

We were able to separate the products of the fibrinogen-fibrin conversion (stopped at about onequarter of the gelation time). The fibrinogen-sized molecules $\left(s_{20, \mathrm{w}}=7 \mathrm{~S}\right)$ had only alanine and tyrosine $N$-terminal residues (no glycine). Fibrin dimer $\left(s_{20, w}=12 S\right)$ had the $N$-terminal pattern of alanine/ glycine/tyrosine residues $(1: 1: 2)$ per 340000 molecular weight. The soluble fibrin polymers larger than fibrin dimer were separated into two rough classifications, $\mathrm{f}_{\mathrm{x}}\left(s_{20, \mathrm{w}}=17 \mathrm{~S}\right)$ and $\mathrm{f}_{\text {void }}\left(s_{20, \mathrm{w}}=23 \mathrm{~S}\right)$. These mixtures of soluble fibrin polymers contained a significant amount of $N$-terminal alanine when isolated at this early stage (one-quarter of the gelation time), showing the existence of intact $A \alpha$-chains within these soluble fibrin polymers.

The fibrin dimer, on the basis of its $N$-terminal content, could only have been composed by the combination of two $(\alpha)(A \alpha)(B \beta)_{2}(\gamma)_{2}$ fibrin monomers, or from the stoichiometric combination of one $(\alpha)_{2}(B \beta)_{2}$ $(\gamma)_{2}$ fibrin monomer and one unaltered fibrinogen $(\mathrm{A} \alpha)_{2}(\mathrm{~B} \beta)_{2}(\gamma)_{2}$. Benabid $e t$ al. (1977) isolated a noncross-linked fibrin dimer (pH 8.5) and found it to have two intact A-fibrinopeptides and four intact B-fibrinopeptides. This is absolute proof that fibrin polymerization begins on incomplete thrombin activation of fibrinogen. The fibrin dimer model that stoichiometrically contains an unaltered fibrinogen molecule can be rejected on rational and experimental grounds. Rationally, if thrombin activation (removal of the fibrinopeptides) is required to expose an active polymerization surface (or to allow the formation of an active surface) in the fibrin monomer, then no such surface would be available on unactivated fibrinogen molecules; therefore there would exist no driving force for these unactivated fibrinogen molecules to participate in the irreversible association reactions we have observed. Also thrombin proteolysis of both $A \alpha$-chains in one fibrinogen in the presence of a huge excess of intact fibrinogens early in the reaction would be required. Another requirement would be that the valency induced by this double proteolysis is completely satisfied by a single contact with an unaltered fibrinogen molecule to yield a dimer that would be electrically neutral and inert. These constraints are difficult to accept in favour of the simple combination of two $(\alpha)(A \alpha)(B \beta)_{2}(\gamma)_{2}$ activated monomers. Experimentally, it has been shown that the soluble fibrin polymers formed under the conditions of Fig. 1, just before the gelation point (when no fibrinogen-sized molecules remain), contain only traces of $N$-terminal alanine, whereas at about one-quarter of the gelation time soluble fibrin polymer molecules contain about 0.4 residue of alanine/340000 molecular weight (Smith \& Bang, 1972; Smith, 1979). These data preclude the possibility for fibrinogen being a stoichiometric co-reactant in the formation of these soluble polymers. Also, experiments have been conducted whereby soluble fibrin polymer fractions (carefully isolated, including all the various molecular size populations, e.g. the fractions shown in Fig. $2 b$ ) and fibrinogen molecules have been incubated together for various periods of time (ranging from $1 \mathrm{~h}$ to 3 days) and the mixtures analysed by gel chromatography. The relative concentrations of fibrinogen and soluble fibrin in the experiments were varied from equimolar to large excesses of either component. The experiments were performed with radioactively labelled fibrinogen mixed with unlabelled soluble fibrin polymers, and with radioactively labelled soluble fibrin mixed with unlabelled fibrinogen. In all experiments analytical gel chromatography showed no interaction between fibrinogen and any of the soluble fibrin polymer molecules (G. F. Smith, unpublished work; Smith \& Craft, 1974).

Therefore I conclude that unaltered fibrinogen is not a component of the stable fibrin dimer or of any soluble fibrin polymer. Accordingly, fibrin polymerization must begin after only one $A \alpha$-chain is activated by thrombin and the first soluble polymer is a dimer formed by the self-association of two $(\alpha)(\mathrm{A} \alpha)(\mathrm{B} \beta)_{2}(\gamma)_{2}$ fibrin monomers.

The mechanism of fibrin polymerization, deduced from the present work, is depicted in Fig. 5.

It is most reasonable to visualize the formation of fibrin dimer as a reciprocal alignment of two fibrin monomers such that their active surfaces (somehow accessible after loss of one A-fibrinopeptide) complementarily interact (utilizing hydrophobic and electrostatic bonding and hydrogen-bonds). This type of complementary or reciprocal association would readily explain the inertness or 'dead-end' nature of the soluble fibrin oligomers, for the active surfaces would be mutually neutralized, leaving only unactivated $A \alpha$-chains (and $B \beta$-chains) exposed for future thrombin activation. Also this type of reciprocal alignment would agree with the elegant finding of Doolittle et al. (1972), who demonstrated that the chains of adjacent fibrin subunits of cross-linked fibrin (cross-linked by factor XIIIa) were reciprocally orientated from the $C$-terminal ends of the $\gamma$-chains.

It has been well established that thrombin removes the A-fibrinopeptides from fibrinogen much faster than the B-fibrinopeptides (Bettleheim, 1956; Laurent \& Blomback, 1958).

Therefore the growth of the soluble fibrin polymers is postulated to involve thrombin activation of one of 


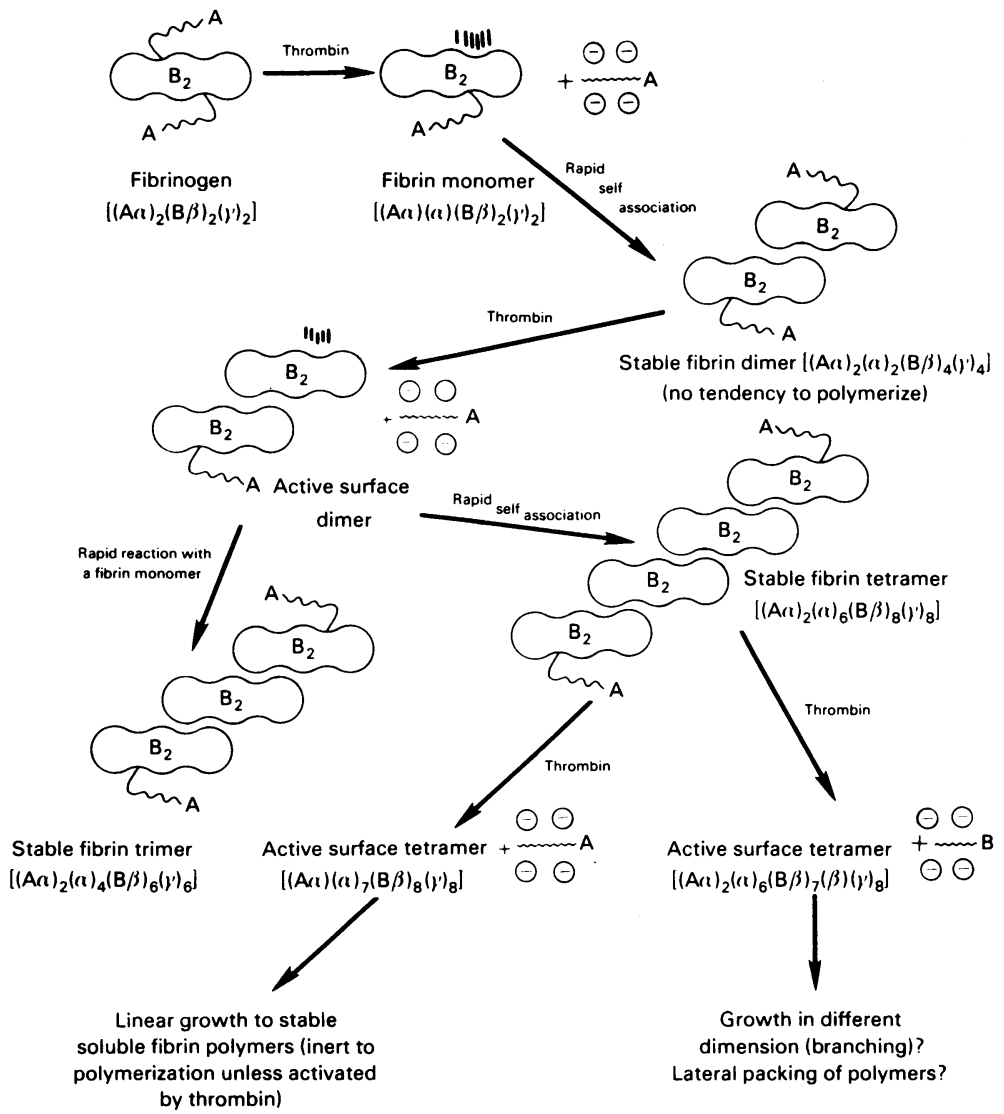

Fig. 5. Fibrin polymerization induced by thrombin

The Figure shows a schematic representation of the mechanism of fibrin polymerization suggested by the findings in the present paper, i.e. the stability of the soluble fibrin polymers, the progressive irreversible fibrin polymer growth, the structure of fibrin dimer and the control exerted by thrombin over the process. Also included in the Figure are the reciprocal alignment of oligomeric subunits (Doolittle et al., 1972), the localization of the fibrinopeptides at the centre portion of the molecule (Blombäck \& Blombäck, 1972) and the faster release of A-fibrinopeptides compared with B-fibrinopeptides (Laurent \& Blombäck, 1958).

the remaining $A \alpha$-chains on the fibrin dimer, followed by rapid reciprocal associations between the activated dimer species and other activated species generated in the thrombin-driven reaction mixture. Each time a reciprocal association occurs, the product of the association (a fibrin oligomer) becomes an inert, or 'dead-ended', molecule, requiring thrombin for further activation (each stable polymer would have an $A \alpha$-chain at either end and a large number of $B \beta$-chains along its length). In this fashion progressively larger fibrin polymers are built up and thrombin is constantly in control over the irreversible process. The condition for gelation is simply the asymmetry of the growing soluble fibrin polymers (Flory, 1972; Nelb et al., 1976), and this condition is known to require only the release of fibrinopeptide A (Laurent \& Blombäck, 1958). The associations of the soluble fibrin polymers into fibrils and the corresponding role of the activation sites liberated by fibrinopeptide B release are beyond the experimental scope of the present paper. These issues have been discussed in the work of Blomback et al. (1978), who suggest that fibrin polymeric structures are a prerequisite for fibrinopeptide B release, and of Shen et al. (1977), who suggest that the fibril-fibre structural dimensions are dependent on fibrinopeptide $B$ release.

The mechanism of fibrin polymerization reported in the present paper suggests that stable fibrin polymers are progressively constructed in an orderly way and that sequential thrombin activation controls the process. This, in turn, suggests that the bulk of thermodynamic, kinetic and physical observations that were made with systems of dispersed clotted fibrin may not be valid, since the reaggregation of 
fully activated subunits can follow pathways not allowed during the sequential activation process. However, the discovery that the soluble fibrin polymers are stable at $\mathrm{pH} 7.4$ and $0.15 \mathrm{M}-\mathrm{NaCl}$ will afford the opportunity to re-examine the fibrinpolymerization reactions from many experimental approaches, under these nearly physiological conditions.

The experimental thrombin concentrations used in the present study were similar to that expected to be generated in blood (Nemerson et al., 1974; Rosenberg et al., 1975). Moreover, it has been shown that these concentrations of thrombin are required to produce fibrin fibres, which are microscopically indistinguishable from fibres isolated from human arterial thrombi (Buchanan et al., 1977).

Also, the stable fibrin-like polymers observed in patients' plasma (Kierulf \& Godal, 1972; Graeff et al., 1973; Donati et al., 1976; Edgar et al., 1977) can be accounted for by this mechanism. Not only is it no longer necessary to explain fibrin solubility by invoking hypothetic fibrinogen-fibrin 'complexes' (Shainoff \& Page, 1960, 1962; Fletcher et al., 1977; Sherman, 1977), but also any such 'complex' hypothesis will have to be justified in the light of the present demonstration that fibrin polymers are soluble and stable under physiological conditions.

The technical assistance of Miss Trelia J. Craft is gratefully acknowledged. The sedimentation velocity data were kindly provided by Mr. Angelo J. Veros and Dr. Bruce H. Frank. $N$-Terminal amino acid analyses were kindly performed by Dr. Gene Rearick and Dr. Marvin Murray, University of Louisville, Louisville, KY, U.S.A. I am grateful to Anna Zimmer, Lillian Witter and Jacqueline Sundboom for helping assemble this manuscript. Dr. William W. Bromer provided invaluable aid in the discussion of basic concepts and problems involved with this report.

\section{References}

Alkjaersig, N., Fletcher, A. \& Burstein, R. (1975) Am. J. Obstet. Gynecol. 122, 199-211

Benabid, Y., Concord, E. \& Suscillon, M. (1977) Thromb. Haemostas. 37, 144-153

Bettleheim, F. R. (1956) Biochim. Biophys. Acta 19, 121-130

Blombäck, B. (1958) Ark. Kemi 12, 321-335

Blombäck, B. \& Blombäck, M. (1972) Ann. N. Y. Acad. Sci. 202, 77-79

Blombäck, B., Hessel, B., Hogg, D. \& Theikildsen, L. (1978) Nature (London) 275, 501-505

Buchanan, J. M., Chen, L. B., Hamazaki, T., Lenk, E. \& Waugh, D. F. (1977) in Chemistry and Biology of Thrombin (Lundblad, R. L., Fenton, J. W. \& Mann, K. G., eds.), pp. 263-273, Ann Arbor Science, Ann Arbor

Davis, B. J. (1964) Ann. N.Y. Acad. Sci. 121, 404-427

Donati, M. B., Venhaeghe, R., Culasso, D. E. \& Vermylen, J. (1976) Thromb. Haemostas. 36, 14-26
Doolittle, R. F. (1973) Adv. Protein Chem. 27, 1-109

Doolittle, R. F., Cassman, K. G., Chen, R., Sharp, J. J. \& Wooding, G. L. (1972) Ann. N.Y. Acad. Sci. 202, 114-126

Edgar, W., McKillop, C., Howie, P. W. \& Prentice, C. R. M. (1977) Thromb. Res. 10, 567-574

Fletcher, A. P., Alkjaersig, N. K., O'Brien, J. R. \& Tulevski, V. (1977) J. Lab. Clin. Med. 89, 1349-1364

Flory, P. J. (1972) in Polymerization in Biological Systems: Ciba Found. Symp. 7, 109-124

Graeff, H., von Hugo, R. \& Hafter, R. (1973) Thromb. Res. 3, 465-476

Kazal, L. A., Amsel, S., Miller, O. P. \& Tocantins, L. M. (1963) Proc. Soc. Exp. Biol. Med. 113, 989-994

Kazal, L. A., Grannis, G. F. \& Tocantins, L. M. (1964) in Blood Coagulation, Hemorrhage and Thrombosis, pp. 232-245, Grune and Stratton, New York

Kierulf, P. \& Abildgaard, U. (1971) Scand. J. Clin. Lab. Invest. 28, 231-240

Kierulf, P. \& Godal, H. C. (1972) Scand. J. Haematd. 9, 370-376

Laurent, T. C. \& Blombäck, B. (1958) Acta Chem. Scand. 12, 1875-1877

Ly, B. \& Jakobsen, E. (1975) Thromb. Res. 6, 65-73

Manyakov, V. F., Varetskaya, T. V. \& Belitser, V. A. (1977) Mol. Biol. 11, 908-913

McCoy, L. E., Walz, D. A. \& Seegers, W. H. (1974) Thromb. Diath. Haemorrh. S57, 126-132

Mosesson, M. (1962) Biochim. Biophys. Acta 57, 204-213

Mosesson, M. \& Sherry, S. (1966) Biochemistry 5, 28292835

Murray, M. \& Smith, G. F. (1968) Anal. Chem. 40, $440-442$

Nelb, G. W., Gerth, C. \& Ferry, J. D. (1976) Biophys. Chem. 5, 377-287

Nemerson, Y., Silverberg, S. A. \& Jesty, J. (1974) Thromb. Diath. Haemorrh. 32, 57-64

Rasmussen, P. S. (1955) Biochim. Biophys. Acta 16, 157-158

Rosenberg, J. S., Beeler, D. L. \& Rosenberg, R. D. (1975) J. Biol. Chem. 250, 1607-1617

Scheraga, H. A. \& Laskowski, M. L., Jr. (1957) $A d v$. Protein Chem. 12, 1-131

Shainoff, J. R. \& Page, I. H. (1960) Circ. Res. 8, 10131022

Shainoff, J. R. \& Page, I. H. (1962) J. Exp. Med. 116, 687-707

Shen, L. L., Hermans, J., McDonagh, J. \& McDonagh, R. P. (1977) Am. J. Physiol. 232, H629-H633

Sherman, L. A. (1977) Thromb. Haemostas. 38, 809-822

Sherry, S., Troll, W. \& Gluek, H. (1954) Physiol. Rev. 34, 736-741

Smith, G. F. (1968) Ph.D. Thesis, University of Louisville, $1-152$

Smith, G. F. (1977) Biochem. Biophys. Res. Commun. 77, 111-117

Smith, G. F. (1979) Abstr. Conf. Am. Chem. Soc., Biol. Chem. Div., Honolulu, abstr. no. 31

Smith, G. F. \& Bang, N. U. (1972) Biochemistry 11, 2958-2966

Smith, G. F. \& Craft, T. J. (1974) Fed. Proc. Fed. Am. Soc. Exp. Biol. 33, 218

Smith, G. F. \& Craft, T. J. (1976) Biochem. Biophys. Res. Commun. 71, 738-745 
Smith, G. F. \& Murray, M. (1968) Anal. Biochem. 23, 183-195

Smith, G. F. \& Murray, M. (1970) Arch. Pathol. 89, 235-246

Svendsen, L., Blombäck, B., Blombäck, M. \& Olsson, P. I. (1972) Thromb. Res. 1, 267-278 von Hugo, R. \& Graeff, H. (1973) Thromb. Res. 3, 183-190 Wessler, S. (1977) Fed. Proc. Fed. Am. Soc. Exp. Biol. 36, 66-69

Zimmerman, T. S. (1976) in Textbook of Immunopathology (Miescher, P. A. \& Muller-Eberhard, H. J., eds.), pp. 95-115, Grune \& Stratten, New York 